\title{
Adaptive Coordinated Path Tracking Control Strategy for Autonomous Vehicles with Direct Yaw Moment Control
}

\author{
Ying Tian ${ }^{1}$, Qiangqiang Yao ${ }^{1 *}$, Peng Hang ${ }^{2}$ and Shengyuan Wang ${ }^{1}$
}

\begin{abstract}
It is a striking fact that the path tracking accuracy of autonomous vehicles based on active front wheel steering is poor under high-speed and large-curvature conditions. In this study, an adaptive path tracking control strategy that coordinates active front wheel steering and direct yaw moment is proposed based on model predictive control algorithm. The recursive least square method with a forgetting factor is used to identify the rear tire cornering stiffness and update the path tracking system prediction model. To adaptively adjust the priorities of path tracking accuracy and vehicle stability, an adaptive strategy based on fuzzy rules is applied to change the weight coefficients in the cost function. An adaptive control strategy for coordinating active front steering and direct yaw moment is proposed to improve the path tracking accuracy under high-speed and large-curvature conditions. To ensure vehicle stability, the sideslip angle, yaw rate and zero moment methods are used to construct optimization constraints based on the model predictive control frame. It is verified through simulation experiments that the proposed adaptive coordinated control strategy can improve the path tracking accuracy and ensure vehicle stability under high-speed and largecurvature conditions.
\end{abstract}

Keywords: Autonomous vehicles, Path tracking, Model predictive control, Adaptive coordinated

\section{Introduction}

The rapid development of artificial intelligence technology, it has brought new opportunities and challenges to autonomous vehicles, which have received increasing attention [1-4]. The main tasks in autonomous vehicle systems include environmental perception, vehicle positioning, behavior decision-making, motion planning, and execution control. As the core task in execution control, path tracking should ensure that the vehicle accurately follows the reference path and remains vehicle stability [5-8].

In active front wheel steering (AFS), steering operations can be applied to correct understeer or oversteer.

\footnotetext{
*Correspondence: yaoqiang@bjtu.edu.cn

${ }^{1}$ Beijing Key Laboratory of Powertrain for New Energy Vehicle, School of Mechanical, Electronic and Control Engineering, Beijing Jiaotong University, Beijing 100044, China

Full list of author information is available at the end of the article
}

AFS has been extensively studied and applied for path tracking, and shown to have little effect on vehicle longitudinal dynamics and ride comfort. In Ref. [9], a switched strategy was proposed and the effects of different error models on the vehicle transient and steady-state characteristics were analyzed. Lyapunov stability arguments were used to exploit the D-stability of vehicle path tracking closed-loop system to improve its transient behaviors [10]. Inspired by a model-free controller, an ultra-local model predictive control strategy for path tracking was proposed in Ref. [11]. To handle time-varying parameters, a robust lateral control strategy based on $H_{\infty}$ control was studied [12]. A dynamic path tracking controller for a double-steering mobile robot on slippery grounds was designed in Ref. [13].

Vehicle limiting control methods to meet vehicle safety requirements under high-speed and large-curvature conditions have received increasing attention. Many control 
algorithms have been employed to improve the controller performance of AFS vehicles under extreme conditions. The center of percussion in the vehicle was used to design steering controller for autonomous racing vehicles in Ref. [14]. The vehicle sideslip angle in the feedback-feedforward control law was introduced in Ref. [15] to improve the path tracking accuracy and stability at the limits of handling. An obstacle avoidance and vehicle stability control strategy using safe envelopes based on model predictive control (MPC) was proposed in Refs. [16, 17]. The control strategy for drifting along the reference path beyond vehicle stability limits was studied in Ref. [18]. However, the controller performance is limited when the tire lateral force approaches the limits of handling.

Direct yaw moment control (DYC) can correct the vehicle posture by using differential braking to generate yaw moment. Coordinated control strategies based on AFS and DYC are therefore widely used for vehicle path tracking. Integrated longitudinal and lateral vehicle stability control was realized using a modular optimal control structure-based MPC, and the tracking errors were minimized using longitudinal control and yaw moment [19]. To enhance vehicle directional stability and steerability, a new integrated control system based on nonlinear MPC was designed to coordinates active torque vectoring and electronic stability control [20]. A robust MPC with finite time horizon for path tracking was studied to address parametric uncertainties and external disturbances [21]. In Ref. [22], path tracking control via second-order sliding mode and nonlinear disturbance observer was studied. The coordinated strategy between path tracking and DYC based on vehicle sideslip angle estimation was proposed in Ref. [23]. In Ref. [24], MPC was combined with sliding surface control to track the reference yaw rate based on coordinated control frame. In the above works, the control strategies was coordinated by DYC throughout the entire process of path tracking, and the advantages of AFS were not fully utilized. Differential braking affected the vehicle longitudinal dynamics and reduced the vehicle speed.

The tire cornering stiffness is an important parameter that reflects the tire dynamics. The tire dynamics tends to be in the nonlinear or saturated regime under high-speed and large-curvature conditions. A robust $H_{\infty}$ output-feedback control method for path tracking was proposed to deal with tire cornering stiffness uncertainty [25]. In Ref. [26], a novel robust adaptive indirect control method based on an exponential-like sliding-mode fuzzy type-2 neural network approach was proposed to enhance the path tracking performance. A robust event-triggered automatic steering control approach with $H_{\infty}$ performance was studied in Ref. [27]. This methods use the bounded disturbance energy hypothesis to handle parameter uncertainties. The choice of the assumed bounded range is important because an inappropriate choice may lead to excessive conservativeness. A parameter identification model for tire cornering stiffness was derived in Ref. [28] based on vehicle dynamics model, and the tire cornering stiffness in the linear segment was measured in low-speed circle test. The sideslip angle was eliminated, and the normalized tire lateral stiffness estimated using the recursive least square method [29]. As the amount of online observation data increased, the identification algorithm became susceptible to dimensional disasters and excessive reference to historical data.

Based on the above previous works, a novel adaptive coordinated path tracking control strategy with direct yaw moment control based on MPC is proposed in this study to improve the tracking accuracy and ensure vehicle stability. The architecture of the adaptive coordinated MPC path tracking control scheme is shown in Figure 1. The main innovations and contributions of this study are as follows: (1) The tire nonlinear dynamic characteristics are described using a linear tire model. An online tire stiffness identification method is proposed in which the least square method with a forgetting factor is used to update the system prediction model parameters to improve the accuracy of vehicle state prediction. (2) To improve the tracking accuracy under high-speed and large-curvature conditions, an adaptive coordinated path tracking control strategy was proposed, the weight coefficient of the objective cost function is changed using fuzzy rules, and adaptive coordination between path tracking and DYC is realized. (3) The adaptive coordinated MPC controller is designed and tested on the CarSim-Simulink platform under these conditions.

The organization of this paper is as follows: The model of the path tracking control system and controller design are presented in Section 2. The adaptive coordinated control strategy is presented in Section 3. The simulation is

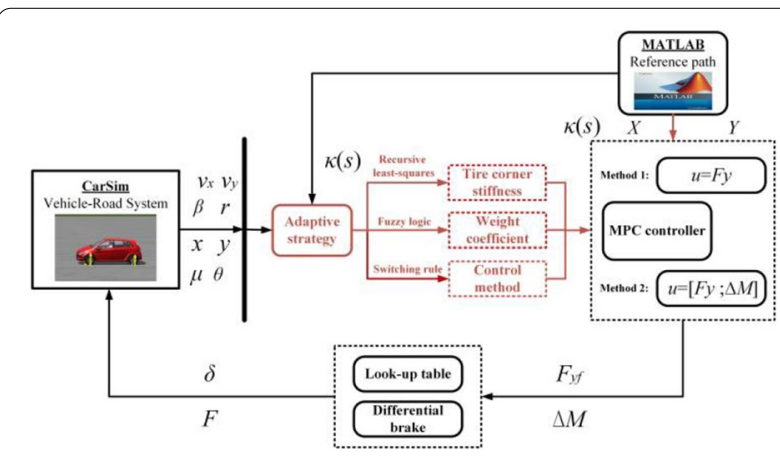

Figure 1 Architecture of adaptive coordinated MPC path tracking control scheme 
presented in Section 4. Section 5 concludes the paper with a brief discussion of the results.

\section{Model of Path Tracking Controller for Autonomous Steering}

\subsection{Vehicle Dynamics Model}

Because the proposed controller requires the vehicle dynamics information under high-speed and large-curvature conditions, the vehicle lateral, yaw, and roll dynamics are established as shown in Figure 2 to capture the essential vehicle dynamics state parameters. The tires are the only components that connect the road to the vehicle. All movements, including the starting, acceleration, braking, and turning of the vehicle, are realized through the tires. During the path tracking process under high-speed and large-curvature conditions, vehicle yaw moment is provided by the lateral force of the front and rear tires. The lateral acceleration of the vehicle is mainly caused by the lateral force of the front and rear tires. At the same time, the coupling relationship between the yaw rate and longitudinal velocity can produce additional vehicle lateral acceleration. The vehicle roll dynamics are described using the characteristics of the suspension damping and stiffness considering the coupling relationship between

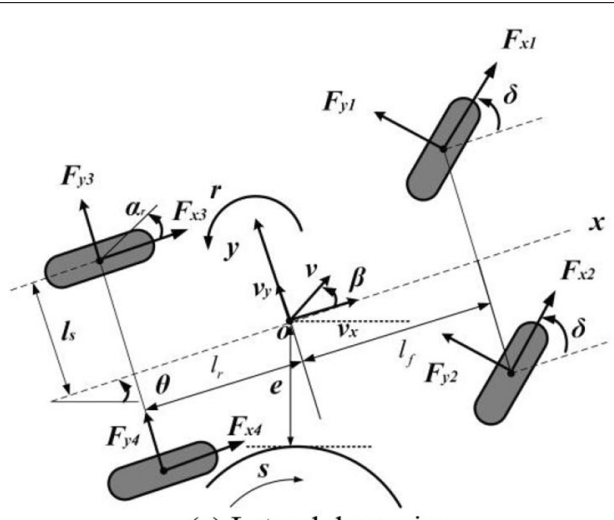

(a) Lateral dynamics

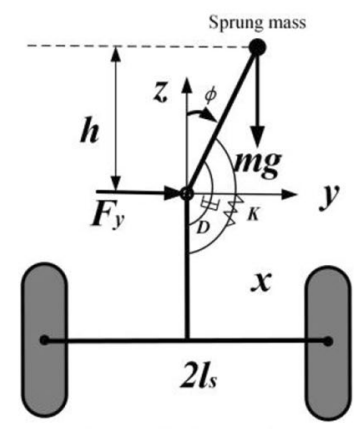

(b) Roll dynamics

Figure 2 Vehicle dynamics model the vehicle lateral velocity, longitudinal velocity, and roll dynamics.

In Figure 2, $m$ is the vehicle total mass. $h$ is the height of the sprung mass from the roll center, $l_{f}$ and $l_{r}$ are the distances from the center of gravity to the front and rear axles, respectively. $l_{s}$ is the half distance between the left and right tires. $g$ is the acceleration due to gravity. $v_{x}$ and $v_{y}$ are the longitudinal and lateral speed, respectively. $r$ and $\phi$ are the yaw rate and roll angle, respectively. $F_{x 1}$ and $F_{x 3}$ are the longitudinal tire force of the front and rear axles. $F_{y 1}$ and $F_{y 3}$ are the lateral tire force of the front and rear axles. $\delta$ and $\beta$ are the front tire steering angle and vehicle sideslip angle. $\theta$ is the vehicle yaw angle. $K$ and $D$ are the suspension roll damping and stiffness.

The vehicle dynamics model is given by Eq. (1).

$$
\left\{\begin{array}{l}
m\left(\dot{v}_{y}+v_{x} r\right)=2 F_{x 1} \sin \delta+2 F_{y 1}+2 F_{y 3}, \\
I_{z} \dot{r}=\left(2 F_{x 1} \sin \delta+2 F_{y 1}\right) l_{f}-2 F_{y 3} l_{r}+M, \\
I_{x} \ddot{\phi}=m_{s} h\left(\dot{v}_{y}+v_{x} r\right)+m_{s} g h \phi-K_{\phi} \phi-D_{\phi} \dot{\phi}, \\
\dot{Y}=v_{x} \sin (\theta)+v_{y} \cos (\theta), \\
\dot{X}=v_{x} \cos (\theta)+v_{y} \sin (\theta),
\end{array}\right.
$$

where $m_{\mathrm{s}}$ is the vehicle sprung mass. $M$ is the external yaw moment. $I_{\mathrm{z}}$ is the moment of inertia about $z$-axis. $I_{x}$ is the moment of inertia of sprung mass about the $x$-axis. $X$ and $Y$ are the coordinate positions of the vehicle in $X O Y$ coordinate system.

Under the small-angle approximation, the vehicle dynamics model can be approximated as

$$
\left\{\begin{aligned}
\dot{v}_{y} & \approx\left(2 F_{y 1}+2 F_{y 3}\right) / m-v_{x} r, \\
\dot{r} & \approx\left(2 F_{y 1} l_{f}-2 F_{y 3} l_{r}+M\right) / I_{z}, \\
\ddot{\phi} & \approx\left[m_{s} h\left(\dot{v}_{y}+v_{x} r\right)+m_{s} g h \phi-K \phi-D \dot{\phi}\right] / I_{x} .
\end{aligned}\right.
$$

\subsection{Tire Dynamics Model}

The Fiala brush tire model is highly accurate and fully accounts for the nonlinear dynamic characteristics. The lateral force of the tire can be described as a function of the tire slip angle.

$$
\begin{aligned}
F_{y[f, r]}= & f_{\text {tire }}(\alpha) \\
= & \left\{\begin{array}{l}
-C_{\alpha} \tan \alpha+\frac{C_{\alpha}^{2}}{3 \mu F_{z}}|\tan \alpha| \tan \alpha \\
-\frac{C_{\alpha}^{3}}{27 \mu^{2} F_{z}^{2}},|\alpha|<\arctan \left(\frac{3 \mu F_{z}}{C_{\alpha}}\right), \\
-\mu F_{z} \operatorname{sgn}(\alpha), \text { otherwise, }
\end{array}\right.
\end{aligned}
$$

where $\alpha$ and $C_{\alpha}$ are the tire slip angle and cornering stiffness, respectively. $\mu$ is the adhesion coefficient between the road surface and the tire. $F_{\mathrm{z}}$ is the vertical force. 
Because the tire slip angle does not directly participate in the control optimization solution process and the vehicle lateral speed is relatively small compared to the longitudinal speed, the tire slip angle of the front and rear tires can be approximated as

$$
\left\{\begin{array}{l}
\alpha_{f}=\arctan \left(\frac{v_{y}+l_{f} r}{v_{x}}\right)-\delta \approx \frac{v_{y}+l_{f} r}{v_{x}}-\delta, \\
\alpha_{r}=\arctan \left(\frac{v_{y}-l_{r} r}{v_{x}}\right) \approx \frac{v_{y}-l_{r} r}{v_{x}} .
\end{array}\right.
$$

To make full use of the tire nonlinear dynamics and reduce the nonlinear complexity of the controller, the front tire lateral force can be considered as the control input for the MPC optimization and mapped to the desired $\delta$ via

$$
\delta=\frac{v_{y}+l_{f} r}{v_{x}}-\alpha_{f} .
$$

The nonlinear dynamics of the rear tire should be properly accounted for to approximate the MPC linear optimization problem accurately. The steady-state solution of the rear lateral force is expressed as

$$
\bar{F}_{r, s s}=\frac{m l_{f}}{L} v_{x}^{2} \kappa,
$$

where $\kappa$ is the curvature of the reference path at the distance $s$.

The equivalent cornering stiffness can be written as

$$
\bar{C}_{r}=\frac{\left(\bar{F}_{r, s s}-\bar{F}_{r}\right)}{\bar{\alpha}_{r, s s}-\bar{\alpha}_{r}},
$$

where $\bar{F}_{r}$ and $\bar{\alpha}_{r}$ are the tire slip angle and corresponding tire lateral force.

The predicted rear tire lateral force in the MPC frame can be written as

$$
F_{y r}=\bar{F}_{r}-\bar{C}_{r}\left(\alpha-\bar{\alpha}_{r}\right) .
$$

\subsection{Path Tracking Model}

It is desired that the path tracking should follow the reference path accurately by minimizing the lateral and heading deviations while maintaining rollover and lateral stability. The relationship between the vehicle and reference path is illustrated in Figure 3, where the $C M$ point represents the center of vehicle mass.

The path tracking kinematic relationship can be expressed as

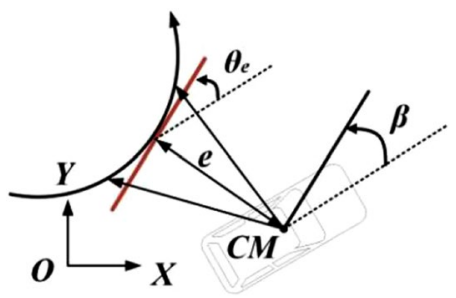

Figure 3 Path tracking kinematic relationship

$$
\left\{\begin{aligned}
\dot{e} & =v_{y}+v_{x} \theta_{e}, \\
\dot{\theta}_{e} & =r-v_{x} \kappa(s), \\
\dot{s} & =v_{x} \cos \left(\theta_{e}\right)-v_{y} \sin \left(\theta_{e}\right), \\
\theta_{e} & =\theta-\theta_{d},
\end{aligned}\right.
$$

where $\theta$ and $\theta_{d}$ are the actual yaw angle and reference yaw angle of vehicle, respectively. $\kappa(s)$ is the curvature of the reference path at the position $s$.

\subsection{Design of Path Tracking Controller Based on MPC}

\subsubsection{Prediction Model}

The controller state space model, in which the front tire lateral force and external yaw moment are taken as the control inputs, can be derived from Eqs. (2), (4), (8), and (9) as

$$
\left\{\begin{array}{l}
\dot{x}=A_{1} x+B_{1} u+E_{1} \kappa+D_{1} \\
y=C_{1} x .
\end{array}\right.
$$

The state vector, system output vector, control input, the state matrix and output matrix are respectively expressed as

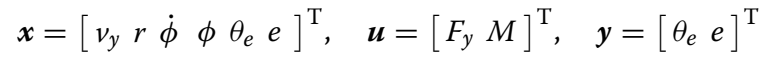

$A_{1}=\left[\begin{array}{cccccc}\frac{-2 \bar{C}_{r}}{m v_{x}} & \frac{2 l_{r} \bar{C}_{r}}{m v_{x}}-v_{x} & 0 & 0 & 0 & 0 \\ \frac{2 l_{r} \bar{C}_{r}}{I_{Z} v_{x}} & -\frac{2 l_{r}^{2} \bar{C}_{r}}{I_{z} v_{x}} & 0 & 0 & 0 & 0 \\ -2 \bar{C}_{r} \Theta_{1} & \frac{2 l_{r} \bar{C}_{r} \Theta_{1}}{v_{x}} & -D \Theta_{2} & \left(m_{s} h g-K\right) \Theta_{2} & 0 & 0 \\ \frac{v_{x}}{0} & 0 & 0 & 1 & 0 & 0 \\ 0 & 1 & 0 & 0 & 0 & 0 \\ 1 & 0 & 0 & 0 & v_{x} & 0\end{array}\right]$

$\boldsymbol{B}_{1}=\left[\begin{array}{cc}0 & \frac{2}{m} \\ \frac{-1}{I_{Z}} & \frac{2 l_{f}}{I_{Z}} \\ 0 & 2 \Theta_{1} \\ 0 & 0 \\ 0 & 0 \\ 0 & 0\end{array}\right], \boldsymbol{E}_{1}=\left[\begin{array}{c}0 \\ 0 \\ 0 \\ 0 \\ -v_{x} \\ 0\end{array}\right], \boldsymbol{D}_{1}=\left[\begin{array}{c}\frac{2\left(\bar{F}_{r}+\bar{C}_{r} \bar{\alpha}_{r}\right)}{m} \\ \frac{-2 l_{r}\left(\bar{F}_{r}+\bar{F}_{r} \bar{\alpha}_{r}\right)}{I_{Z}} \\ 2\left(\bar{F}_{r}+\bar{C}_{r} \bar{\alpha}_{r}\right) \Theta_{1} \\ 0 \\ 0 \\ 0\end{array}\right]$

$C_{1}=\left[\begin{array}{llllll}0 & 0 & 0 & 0 & 1 & 0 \\ 0 & 0 & 0 & 0 & 0 & 1\end{array}\right]$ 


$$
\Theta_{1}=\frac{m_{s} h}{m I_{x}+m m_{s} h^{2}}, \Theta_{2}=\frac{1}{I_{x}+m_{s} h^{2}} .
$$

To facilitate controller design and implementation, the continuous-time state-space model is converted into a discrete increment system using previous Euler method and expressed as Eq. (11).

$$
\left\{\begin{aligned}
\xi(k+1) & =\boldsymbol{A} \xi(k)+\boldsymbol{B} \Delta \boldsymbol{u}+\boldsymbol{E} \boldsymbol{\kappa}+\boldsymbol{D}, \\
\eta(k) & =\boldsymbol{C} \xi(k),
\end{aligned}\right.
$$

where $T$ is the sampling time, and

$$
\begin{aligned}
& \boldsymbol{\xi}(k)=[\boldsymbol{x}(k) \boldsymbol{u}(k-1)]^{\mathrm{T}}, \boldsymbol{\eta}(k)=\left[\theta_{e} e\right]^{\mathrm{T}}, \\
& \boldsymbol{A}=\left[\begin{array}{cc}
I+T \boldsymbol{A}_{1} & T \boldsymbol{B}_{1} \\
\mathbf{0} & \boldsymbol{I}
\end{array}\right], \boldsymbol{B}=\left[\begin{array}{c}
T \boldsymbol{B}_{1} \\
\boldsymbol{I}
\end{array}\right]^{\mathrm{T}}, \\
& \boldsymbol{E}=\left[T \boldsymbol{E}_{1} \mathbf{0}\right]^{\mathrm{T}}, \boldsymbol{D}=\left[\begin{array}{c}
T \boldsymbol{D}_{1} \\
\mathbf{0}
\end{array}\right]^{\mathrm{T}}, \\
& C=\left[\begin{array}{lllllll}
0 & 0 & 0 & 0 & 1 & 0 & 0 \\
0 & 0 & 0 & 0 & 0 & 1 & 0
\end{array}\right] \text {. }
\end{aligned}
$$

To guarantee a convex optimization solution and reduce the computational complexity of the MPC, the following assumptions are made

$$
\begin{aligned}
& \boldsymbol{A}_{i, k}=\boldsymbol{A}_{k, k}, i=1, \ldots, i+N_{p}-1, \\
& \boldsymbol{B}_{i, k}=\boldsymbol{B}_{k, k}, i=1, \ldots, i+N_{p}-1,
\end{aligned}
$$

where $N_{p}$ denotes the prediction horizon.

Therefore, the system prediction outputs in the prediction horizon can be expressed as

$$
\boldsymbol{Y}(k)=\boldsymbol{\Psi} \xi(k \mid k)+\boldsymbol{\Phi} \Delta \boldsymbol{u}(k)+\boldsymbol{\Pi},
$$

where $N_{\mathrm{c}}$ denotes the control horizon, and

$$
\begin{gathered}
\boldsymbol{Y}(k)=\left[\begin{array}{c}
\boldsymbol{\eta}(k+1 \mid k) \\
\boldsymbol{\eta}(k+2 \mid k) \\
\vdots \\
\eta\left(k+N_{p} \mid k\right)
\end{array}\right], \boldsymbol{\Psi}=\left[\begin{array}{c}
\boldsymbol{C A} \\
\boldsymbol{C} \boldsymbol{A}^{2} \\
\vdots \\
\boldsymbol{C A}^{N_{P}}
\end{array}\right], \\
\boldsymbol{\Pi}=\left[\begin{array}{c}
\boldsymbol{E} \kappa+\boldsymbol{D} \\
\boldsymbol{A}(\boldsymbol{E} \kappa+\boldsymbol{D})+\boldsymbol{D} \\
\vdots \\
\boldsymbol{A}^{N p-1} \boldsymbol{E} \kappa+\boldsymbol{A}^{N p-1} \boldsymbol{D}+\cdots+\boldsymbol{A D}+\boldsymbol{D}
\end{array}\right] \\
\boldsymbol{\Phi}=\left[\begin{array}{cccc}
\boldsymbol{C} \boldsymbol{B} & 0 & 0 & 0 \\
\boldsymbol{C A B} & \boldsymbol{C} \boldsymbol{B} & 0 & 0 \\
\vdots & \vdots & \ddots & \vdots \\
\boldsymbol{C} \boldsymbol{A}^{N c-1} \boldsymbol{B} & \boldsymbol{C} \boldsymbol{A}^{N c-2} \boldsymbol{B} & \cdots & \boldsymbol{C B} \\
\boldsymbol{C} \boldsymbol{A}^{N c} \boldsymbol{B} & \boldsymbol{C A}^{N c-1} \boldsymbol{B} & \cdots & \boldsymbol{C} \boldsymbol{A} \boldsymbol{B} \\
\vdots & \vdots & \ddots & \vdots \\
\boldsymbol{C} \boldsymbol{A}^{N p-1} \boldsymbol{B} & \boldsymbol{C A}^{N p-2} \boldsymbol{B} & \cdots & \boldsymbol{C A}^{N p-N c-1} \boldsymbol{B}
\end{array}\right]
\end{gathered}
$$

\subsubsection{Objective Cost Function and Optimization}

The path tracking control objective of the MPC controller is the solution of a convex optimization problem at each sampling step. The first optimal value of the control sequence is used as the future input. The objective function and constraints can be expressed as

$$
\begin{aligned}
& \min _{\boldsymbol{u}(k)} J_{N p}=\sum_{i=k}^{k+N p-1} \boldsymbol{\eta}^{\mathrm{T}} \boldsymbol{Q} \boldsymbol{\eta}+\sum_{i=k}^{k+N c-1} \Delta \boldsymbol{u}^{\mathrm{T}} \boldsymbol{R} \Delta \boldsymbol{u}+W \varepsilon, \\
& \text { s.t. }\left[\boldsymbol{H}_{\mathrm{v}} \boldsymbol{x}^{i}\right] \leq \boldsymbol{G}_{\mathrm{v}}, i=0,1, \cdots, N_{p}-1, \\
& -l_{s} \leq y_{Z M P} \leq l_{s}, i=0,1, \cdots, N_{p}-1, \\
& |\Delta \boldsymbol{u}(k+i)| \leq \Delta \boldsymbol{u}_{\max }, i=0,1, \cdots, N_{c}-1, \\
& \Delta \boldsymbol{u}(k+i)=0, i=N_{c}, N_{c}+1, \cdots, N_{p}-1, \\
& |\boldsymbol{u}(k+i)| \leq \boldsymbol{u}_{\max }, i=0,1, \cdots, N_{p}-1,
\end{aligned}
$$

where $\varepsilon$ is a non-negative slack variable used to ensure that the optimization problem is always feasible. The optimal front lateral force input $F_{y f}$ and the external yaw moment $M$ can be obtained using a look-up table and yaw moment allocation algorithm. To accurately capture the propagation of $\beta, r$ and $\phi$ at high frequency, the sampling time $T$ is set to $0.02 \mathrm{~s}$ considering the accuracy of the captured vehicle information. Note that the values of the control horizon $N_{c}$ and prediction horizon $N_{p}$ are set to $N_{c}=20$ and $N_{p}=30$ based on control accuracy and calculation efficiency considerations. The weighting matrices are obtained by iteratively tuning, $R=\operatorname{diag}\{10,1\}$, $Q=\operatorname{diag}\{1000,5\}$, and $W=10$.

\subsubsection{Stability Constraints}

The vehicle lateral stability constraints are defined by the bounds of the two key variables of vehicle sideslip angle $\beta$ and yaw rate $r$. The bounds of the $\beta$ and $r$ reflect the maximum capabilities of the tire under the assumptions of steady-state cornering [30]. The maximum steady-state yaw rate and vehicle sideslip angle can be expressed as

$$
\left\{\begin{array}{l}
r_{\max }=\frac{\mu g}{v_{x}}, \\
\beta_{\max }=\arctan (0.02 \mu g) .
\end{array}\right.
$$

Considering the tire saturation and maximum tire lateral force, the maximum steady-state yaw rate and vehicle sideslip angle can also be expressed as 


$$
\left\{\begin{aligned}
\alpha_{r, s a t} & =\arctan \left(3 l_{f} \mu m g / L C_{a_{r}}\right), \\
r_{\text {max }} & =\mu g / \nu_{x} \\
\beta_{\text {max }} & =\alpha_{r, s a t}+l_{r} r / \nu_{x}
\end{aligned}\right.
$$

The vehicle stability constraints defined by Eq. (20) can be expressed as the matrix inequality.

$$
\left|\boldsymbol{H}_{V} \boldsymbol{x}\right| \leq \boldsymbol{G}_{V}
$$

where

$\boldsymbol{G}_{V}=\left[\begin{array}{ll}\alpha_{r, s a t} & \mu g / \nu_{x}\end{array}\right]^{\mathrm{T}}$.

$$
\boldsymbol{H}_{V}=\left[\begin{array}{ccccccc}
1 / \nu_{x} & -l_{r} / v_{x} & 0 & 0 & 0 & 0 \\
0 & 1 & 0 & 0 & 0 & 0
\end{array}\right] \text {, }
$$

Vehicle rollover stability is a significant concern in transportation design. In particular, vehicle rollover stability is especially important under high-speed and large-curvature extreme conditions. To predict the vehicle rollover propensity, the zero-moment point (ZMP) metric is applied to the projected vehicle trajectories. The ZMP is given by Ref. [31].

$$
y_{Z M P}=h \phi+\frac{h}{g}\left(\dot{v}_{y}+v_{x} r\right)-\frac{I_{x}}{m g} \ddot{\phi} .
$$

According to the state space model, the ZMP can be written as

$$
y_{Z M P}=N_{1} \dot{x}+N_{2} x,
$$

where

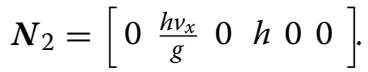

$$
N_{1}=\left[\begin{array}{llllll}
\frac{h}{g} & 0 & -\frac{I_{x}}{m g} & 0 & 0 & 0
\end{array}\right]
$$

The vehicle rollover stability constraints defined by Eq. (23) can be expressed as Eq. (24).

$$
-l_{s} \leq y_{Z M P} \leq l_{s}
$$

\section{Adaptive Coordinated Control for Path Tracking}

When the vehicle is under the extreme conditions of high speed and large-curvature path, the tire slip angle is strictly restricted in the method proposed in Refs. [7, 9] to ensure vehicle stability. This results in a steady state steering phenomenon with poor path tracking control performance in which the steering states remain stable. Therefore, an adaptive coordinated control strategy is proposed here in which the tire cornering stiffness is identified online, the weight coefficient of the objective cost function is adaptively changed, and the yaw moment control is adaptively coordinated to improve the vehicle path tracking performance and ensure vehicle stability under extreme conditions.

\subsection{Tire Cornering Stiffness Identification Based on Recursive Least Square}

To reduce the computational burden of the path tracking controller based on model predictive control, a continuous linearized tire model is used to establish the predictive model. To account for the nonlinear tire dynamic characteristics, a control strategy is proposed based on the optimal front tire lateral force. However, because errors for the lateral force of the rear tire from the model linearization are still present under high-speed and large-curvature conditions, the recursive least square method with a forgetting factor is used to determine the rear tire cornering stiffness.

\subsubsection{Recursive Model}

A simplified two-degrees of freedom (DOF) vehicle dynamics model is established to determine the tire cornering stiffness, as illustrated in Figure 4.

Under the small-angle assumption approximation, the vehicle dynamics model can be expressed as

$$
\left\{\begin{array}{l}
\dot{\beta}=\frac{2 C_{r}+2 C_{f}}{m v_{x}} \beta-\left(\frac{2 l_{r} C_{r}-2 l_{f} C_{f}}{m v_{x}^{2}}+1\right) r+\frac{2 C_{f}}{m v_{x}} \delta_{f}, \\
\dot{r}=\frac{-2 l_{r} C_{r}+2 l_{f} C_{f}}{I_{z}} \beta+\frac{-2 l_{r}^{2} C_{r}-2 l_{f}^{2} C_{f}}{I_{z} v_{x}} r+\frac{2 l_{f} C_{f}}{I_{z}} \delta_{f} .
\end{array}\right.
$$

The vehicle dynamics equation can be discretized using the previous Euler method and expressed as

$$
\left\{\begin{array}{l}
\beta(k+1)-\beta(k)=\frac{2 C_{r}+2 C_{f}}{m v_{x}} T \beta(k), \\
-\left(\frac{2 l_{r} C_{r}-2 l_{f} C_{f}}{m v_{x}^{2}}+1\right) \operatorname{Tr}(k)+\frac{2 C_{f}}{m v_{x}} T \delta_{f}(k), \\
r(k+1)-r(k)=\frac{-2 l_{r} C_{r}+2 l_{f} C_{f}}{I_{z}} T \beta(k), \\
+\frac{-2 l_{r}^{2} C_{r}-2 l_{f}^{2} C_{f}}{I_{z} v_{x}} \operatorname{Tr}(k)+\frac{2 l_{f} C_{f}}{I_{z}} T \delta_{f}(k) .
\end{array}\right.
$$

Considering the vehicle data for the first $k$ steps and transforming the above equation into the least squares form, the recursive model of the vehicle tire cornering stiffness can be expressed as

$$
\boldsymbol{M}(k) \hat{\boldsymbol{C}}=\boldsymbol{N}(k)
$$

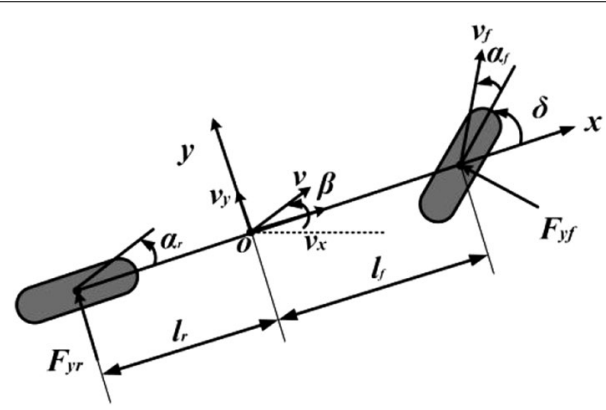

Figure 4 2-DOF vehicle dynamics model 
where the matrixes $\boldsymbol{M}(k)$ and $\boldsymbol{N}(k)$ are given by

$$
\begin{gathered}
\boldsymbol{M}(k)=\left[\begin{array}{cc}
\frac{2 T \beta(1)+2 T \delta_{f}(1)}{m v_{x}}+\frac{2 l_{f} r(1)}{m v_{x}^{2}} & \frac{2 T \beta(1)}{m v_{x}}-\frac{2 l_{r} \operatorname{Tr}(1)}{m v_{x}^{2}} \\
\frac{2 l_{f} T \beta(1)+2 T \delta_{f}(1)}{I_{z}}-\frac{2 l_{f}^{2} \operatorname{Tr}(1)}{I_{z} v_{x}} & \frac{-2 l_{r} T \beta(1)}{I_{z}}-\frac{2 l_{r}^{2} \operatorname{Tr}(1)}{I_{z} v_{x}} \\
\vdots & \vdots \\
\frac{2 T \beta(k)+2 T \delta_{f}(k)}{m v_{x}}+\frac{2 l_{f} r(k)}{m v_{x}^{2}} & \frac{2 T \beta(k)}{m v_{x}}-\frac{2 l_{r} \operatorname{Tr}(k)}{m v_{x}^{2}} \\
\frac{2 l_{f} T \beta(k)+2 T \delta_{f}(k)}{I_{z}}-\frac{2 l_{f}^{2} \operatorname{Tr}(k)}{I_{z} v_{x}} & \frac{-2 l_{r} T \beta(k)}{I_{z}}-\frac{2 l_{r}^{2} \operatorname{Tr}(k)}{I_{z} v_{x}}
\end{array}\right], \\
\boldsymbol{N}(k)=\left[\begin{array}{c}
\beta(2)-\beta(1)+\operatorname{Tr}(1) \\
r(2)-r(1) \\
\vdots \\
\beta(k+1)-\beta(k)+\operatorname{Tr}(k) \\
r(k+1)-r(k)
\end{array}\right], \hat{\boldsymbol{C}}=\left[\begin{array}{c}
\hat{C}_{f} \\
\hat{C}_{r}
\end{array}\right] .
\end{gathered}
$$

\subsubsection{Recursive Least Square Method with Forgetting Factor}

The recursive least square method is modified to provide new parameter identification based on the previous steps. However, the parameter identification of the tire cornering stiffness may become inaccurate because of the data saturation phenomenon. Therefore, a forgetting factor is introduced to overcome the problem of data saturation by reducing the impact of old data. The recursive model can be rewritten as

$$
\bar{M}(k) \hat{C}=\bar{N}(k),
$$

where

$$
\begin{aligned}
\overline{\boldsymbol{M}}(k) & =\left[\begin{array}{c}
\lambda \boldsymbol{\alpha}(k-1) \\
\boldsymbol{\alpha}(k)
\end{array}\right], \overline{\boldsymbol{N}}(k)=\left[\begin{array}{c}
\lambda \boldsymbol{\gamma}(k-1) \\
\boldsymbol{\gamma}(k)
\end{array}\right], \\
\boldsymbol{\gamma}(k) & =\left[\begin{array}{c}
\beta(k+1)-\beta(k)+\operatorname{Tr}(k) \\
r(k+1)-r(k)
\end{array}\right], \\
\boldsymbol{\alpha}(k) & =\left[\begin{array}{cc}
\frac{2 T \beta(k)+2 T \delta_{f}(k)}{m v_{x}}+\frac{2 l_{f} r(k)}{m v_{x}^{2}} & \frac{2 T \beta(k)}{m v_{x}}-\frac{2 l_{r} \operatorname{Tr}(k)}{m v_{x}^{2}} \\
\frac{2 l_{f} T \beta(k)+2 T \delta_{f}(k)}{I_{z}}-\frac{2 l_{f}^{2} \operatorname{Tr}(k)}{I_{z} v_{x}} & \frac{-2 l_{r} T \beta(k)}{I_{z}}-\frac{2 l_{r}^{2} \operatorname{Tr}(k)}{I_{z} v_{x}}
\end{array}\right]
\end{aligned}
$$

and $\lambda$ is the forgetting factor, which is set to the value of 0.98 .

The cornering stiffness at the $k$ th sampling moment is then given by

$$
\hat{\boldsymbol{C}}=\left[\begin{array}{c}
\hat{C}_{f} \\
\hat{C}_{r}
\end{array}\right]=\left(\overline{\boldsymbol{M}}(k)^{T} \overline{\boldsymbol{M}}(k)\right)^{-1} \overline{\boldsymbol{M}}(k)^{T} \overline{\boldsymbol{N}}(k) .
$$

The tire cornering stiffness at the next moment is identified by updating the prediction model of the vehicle path tracking system with the current parameter identification

$$
\hat{\boldsymbol{C}}(k+1)=\boldsymbol{P}(k+1) \boldsymbol{H}(k+1),
$$

$$
\begin{aligned}
& \boldsymbol{P}(k+1)^{-1}=\overline{\boldsymbol{M}}(k+1)^{\mathrm{T}} \overline{\boldsymbol{M}}(k+1), \\
& \boldsymbol{H}(k+1)=\overline{\boldsymbol{M}}(k+1)^{\mathrm{T}} \overline{\boldsymbol{N}}(k+1) .
\end{aligned}
$$

\subsection{Adaptive Strategy of Weight Coefficients Based on Fuzzy Rule}

To ensure high tracking accuracy and improve vehicle stability, an adaptive strategy for the weight coefficients is applied based on fuzzy rules. Based on the components of the objective cost function, the lateral deviation, heading deviation ratio, and vehicle sideslip angle ratios are chosen as the fuzzy control inputs, which can be expressed as

$$
\left\{\begin{array}{l}
I_{e}=\mathrm{abs}\left(\frac{e_{\text {real }}}{e_{\text {max }}}\right), \\
I_{\theta}=\mathrm{abs}\left(\frac{\theta_{\text {real }}}{\theta_{\text {max }}}\right), \\
I_{\beta}=\mathrm{abs}\left(\frac{\beta_{\text {real }}}{\beta_{\text {max }}}\right) .
\end{array}\right.
$$

The weight coefficients of each index in the objective cost function are then adaptively changed, and the priorities for tracking performance and vehicle stability adjusted. The inputs of the fuzzy controller can be fuzzified into the five fuzzy sets of $\mathrm{ZO}$ (zero), PSr (positive smaller), PS (positive small), PB (positive big), and $\mathrm{PBr}$ (positive larger). The lateral and heading deviations, and the vehicle stability performance weight ratios (sideslip angle, yaw rate, and roll angle) are similarly chosen as the fuzzy control outputs and fuzzified into the four fuzzy sets of $\mathrm{ZO}$ (zero), PS (positive small), PM (positive medium), and PB (positive big).

The correlations between the inputs and outputs in the fuzzy rules are constructed based on driving experience and the consideration of both tracking accuracy and ride comfort when tracking the predetermined path, and listed in Tables 1, 2, 3.

\subsection{Coordinated Strategy for Direct Yaw Moment}

Active front wheel steering is an effective means of ensuring the lateral stability of the vehicle. However, it may lead to understeering under high-speed and large-curvature conditions. DYC can give full play to the vehicle yaw dynamics and has a significant effect on restraining vehicle understeer. Therefore, an adaptive control strategy for coordinating active steering with direct yaw moment under high-speed and largecurvature conditions is proposed to improve tracking performance, as shown in Figure 5. 
Table 1 Lateral deviation fuzzy rules for weight ratio

\begin{tabular}{|c|c|c|c|c|c|c|}
\hline \multirow[t]{2}{*}{$Q_{e}$} & & \multicolumn{5}{|l|}{$I_{e}$} \\
\hline & & ZO & PSr & PS & PB & $\mathrm{PBr}$ \\
\hline \multirow[t]{5}{*}{$I_{\theta}$} & $\mathrm{ZO}$ & $\mathrm{ZO}$ & PS & PS & PM & PB \\
\hline & PSr & $\mathrm{ZO}$ & PS & PS & PM & PB \\
\hline & PS & ZO & PS & PS & PM & PB \\
\hline & PB & $\mathrm{ZO}$ & PS & PS & PM & PB \\
\hline & $\mathrm{PBr}$ & $\mathrm{ZO}$ & PS & PS & PM & PB \\
\hline
\end{tabular}

Table 2 Heading deviation fuzzy rules for weight ratio

\begin{tabular}{|c|c|c|c|c|c|c|}
\hline \multirow[t]{2}{*}{$Q_{\theta}$} & & \multicolumn{5}{|l|}{$I_{e}$} \\
\hline & & ZO & PSr & PS & PB & $\mathrm{PBr}$ \\
\hline \multirow[t]{5}{*}{$I_{\theta}$} & $\mathrm{ZO}$ & $\mathrm{ZO}$ & PS & PS & PM & PB \\
\hline & PSr & ZO & PS & PS & PM & PB \\
\hline & PS & ZO & PS & PS & PM & PB \\
\hline & PB & $\mathrm{ZO}$ & PS & PS & PM & PB \\
\hline & $\mathrm{PBr}$ & $\mathrm{ZO}$ & PS & PS & PM & PB \\
\hline
\end{tabular}

Table 3 Vehicle stability fuzzy rules for weight ratio

\begin{tabular}{|c|c|c|c|c|c|c|}
\hline \multirow[t]{2}{*}{$Q_{\beta}$} & & \multicolumn{5}{|l|}{$I_{e}$} \\
\hline & & zo & PSr & PS & PB & $\mathrm{PBr}$ \\
\hline \multirow[t]{5}{*}{$I_{\beta}$} & $\mathrm{ZO}$ & $\mathrm{ZO}$ & PS & PS & PM & PB \\
\hline & PSr & $\mathrm{ZO}$ & PS & PS & PM & PB \\
\hline & PS & $\mathrm{ZO}$ & PS & PS & PM & PB \\
\hline & PB & $\mathrm{ZO}$ & PS & PS & PM & PB \\
\hline & $\mathrm{PBr}$ & $\mathrm{ZO}$ & PS & PS & PM & PB \\
\hline
\end{tabular}

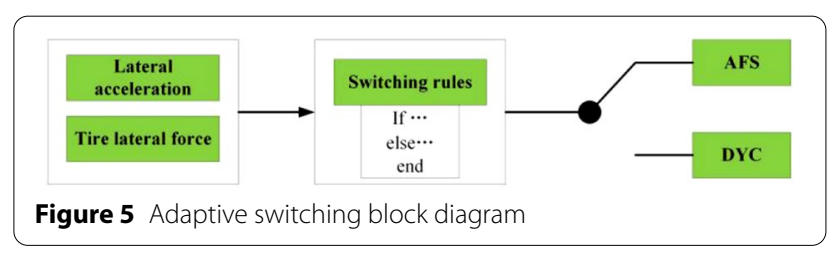

\subsubsection{Analysis of Vehicle Steady State Steering}

As shown in Figure 6, when the autonomous vehicle completes steady-state steering with the maximum steering ability, the lateral force of the front tires and lateral acceleration should satisfy Eq. (34).

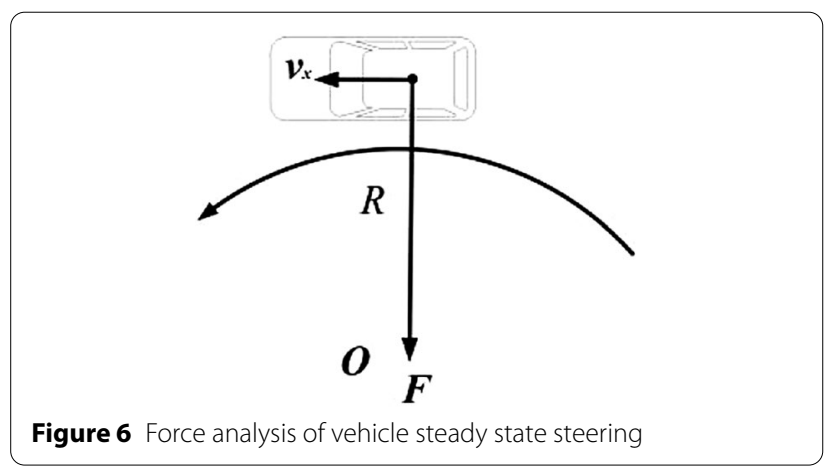

$\left\{\begin{array}{l}F_{y} \leq F_{\text {max }}^{s s} \\ a_{y} \leq a_{\text {max }}^{s s}\end{array}\right.$ 
Under steady state steering conditions, the lateral force of the vehicle tires can be expressed as

$$
\left\{\begin{aligned}
F_{y f}^{s s} & =\frac{m b}{2 L R} v_{x}^{2} \\
F_{y r}^{s s} & =\frac{m a}{2 L R} v_{x}^{2} .
\end{aligned}\right.
$$

where $R$ is the turning radius.

The vehicle motion under steady-state steering motion is approximated as uniform circular motion in the sampling period. The required centripetal acceleration can then be expressed as

$$
\left\{\begin{array}{c}
F=m a_{y}^{s s}, \\
a_{y}^{s s}=\frac{v_{x}^{2}}{R},
\end{array}\right.
$$

Based on the above analysis, the lateral acceleration and lateral force of the tires are chosen as performance indicators for triggering the switching rule.

\subsubsection{Adaptive Switching Rules}

When the switching rules are not triggered, active front wheel steering control is chosen for path tracking. The switching rules are as follows:

1. At time $k$, calculate vehicle reference lateral acceleration.

$$
\begin{aligned}
& \text { if }\left(\left|F_{y}\right| \geq\left|F_{y f}^{s s}-\tau\right| \text { and }\left|a_{y}\right| \geq\left|a_{y}^{s s}\right|\right. \\
& Q_{M}=1
\end{aligned}
$$

Update control matrix and objective cost function;

$$
\boldsymbol{B}_{1}=\left[\begin{array}{cccccc}
\frac{2}{m} & \frac{2 l_{f}}{I_{Z}} & 2 \Theta_{1} & 0 & 0 & 0 \\
0 & \frac{-1}{I_{Z}} & 0 & 0 & 0 & 0
\end{array}\right]^{\mathrm{T}},
$$

else

$Q_{M}=0$

Update control matrix and objective cost function;

$$
\boldsymbol{B}_{1}=\left[\begin{array}{cccccc}
\frac{2}{m} & \frac{2 l_{f}}{I_{Z}} & 2 \Theta_{1} & 0 & 0 & 0 \\
0 & 0 & 0 & 0 & 0 & 0
\end{array}\right]^{\mathrm{T}},
$$

End.
2. Optimization: Find the optimal $\boldsymbol{U}(k)=\left[\begin{array}{ll}F_{\mathrm{y}} & M\end{array}\right]^{\mathrm{T}}$ $\left(\boldsymbol{U}(k)=F_{\mathrm{y}}\right)$.

3. Set $k=k+1$, and update system states and state space model.

In the above, $Q_{M}$ is the weight coefficient of the external yaw moment, $\tau$ is the buffer factor, which is set to the value of 5 .

When the vehicle exits the coordinated control of active front wheel steering and direct yaw moment, the external yaw moment is not directly returned to zero to avoid a sudden change caused by updating the control quantity weight and control matrix. The external yaw moment instead satisfies the following relationship.

$$
M(k)=M_{\max }-\Delta M T k,
$$

where $\Delta M$ is maximum change of yaw moment, and $T$ is the sampling time.

When the vehicle is on a small-curvature path, the AFS controller working mode is adopted, and the controller outputs the desired front tire lateral force. When the vehicle is on a large-curvature path, the coordinated yaw moment control starts to work, and the AFS + DYC controller is adopted. The controller outputs the desired front tire lateral force and external yaw moment. This controller is superior because it can reduce the vehicle yaw rate (shown in Eq. (1)) and thereby reduce the heading and lateral position deviations (shown in Eq. (9)).

\subsection{External Yaw Moment Distribution}

The external yaw moment $M$ generated by the MPC controller should be allocated to the four tires. The lateral force of the front tires is saturated. Therefore, differential braking of the rear tires is used to generate the desired external yaw moment. This can be translated into a multi-objective optimization problem in which a quadratic function is applied to establish the objective cost function for achieving the desired yaw moment with the minimum tire force. The objective function and constraints can be expressed as

$$
\begin{aligned}
& \min J_{1}=\min \frac{1}{2}\left(\boldsymbol{F}_{x}+\boldsymbol{c}\right)^{\mathrm{T}} \boldsymbol{Q}_{1}\left(\boldsymbol{F}_{x}+\boldsymbol{c}\right), \\
& \text { s.t. } \boldsymbol{W}_{F} \boldsymbol{F}_{x}=M, \\
& \boldsymbol{F}_{x, \min } \leq \boldsymbol{F}_{x} \leq \boldsymbol{F}_{x, \max }, \\
& \boldsymbol{W}_{F}=\left[\begin{array}{ll}
-l_{s} & l_{s}
\end{array}\right] \quad \boldsymbol{F}_{x}=\left[\begin{array}{ll}
\boldsymbol{F}_{x 3} & \boldsymbol{F}_{x 4}
\end{array}\right]^{\mathrm{T}},
\end{aligned}
$$

where $Q_{1}=\operatorname{diag}\{1,1\}$ is a weighting positive-definite diagonal matrix and $\boldsymbol{c}=\left[\begin{array}{ll}2 & 2\end{array}\right]^{\mathrm{T}}$ is an offset vector. 
Table 4 The main vehicle parameters

\begin{tabular}{ll}
\hline Parameter & Value \\
\hline Vehicle mass $m(\mathrm{~kg})$ & 1610 \\
Vehicle sprung mass $m_{s}(\mathrm{~kg})$ & 1430 \\
Yaw inertia $I_{Z}\left(\mathrm{~kg} \cdot \mathrm{m}^{2}\right)$ & 2059.2 \\
Roll inertia $I_{x}\left(\mathrm{~kg} \cdot \mathrm{m}^{2}\right)$ & 700.7 \\
Distance from front axle to gravity center $I_{f}(\mathrm{~m})$ & 1.05 \\
Distance from rear axle to gravity center $I_{r}(\mathrm{~m})$ & 1.61 \\
Distance between left and right tires $2 I_{s}(\mathrm{~m})$ & 1.565 \\
Height of the sprung mass from the roll center $h(\mathrm{~m})$ & 0.65 \\
Front cornering stiffness $C_{f}(\mathrm{~N} / \mathrm{rad})$ & 67862 \\
Rear cornering stiffness $C_{r}(\mathrm{~N} / \mathrm{rad})$ & 45714 \\
Suspension roll damping $K(\mathrm{~N} \cdot \mathrm{m} / \mathrm{rad})$ & 145330 \\
Suspension roll stiffness $D(\mathrm{~N} \cdot \mathrm{m} \cdot \mathrm{s} / \mathrm{rad})$ & 4500 \\
Friction coefficient $\mu$ & 0.85 \\
\hline
\end{tabular}

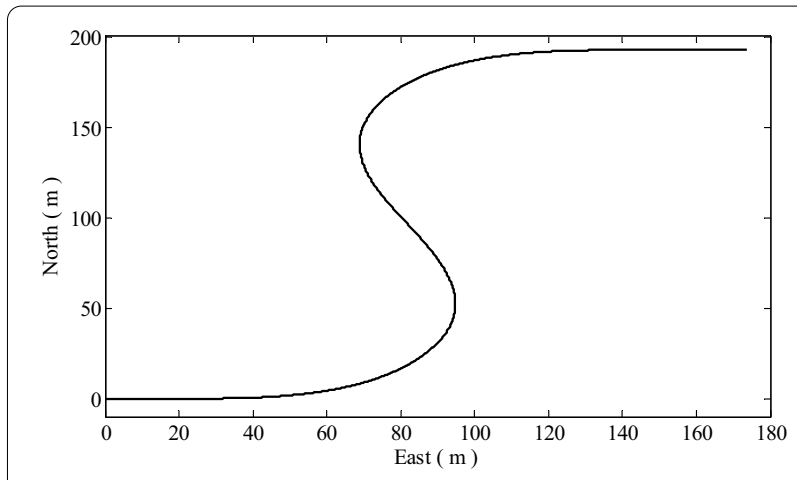

Figure 7 Reference path

\section{Simulation}

\subsection{Reference Path and Vehicle Parameters}

The proposed adaptive coordinated MPC controller was verified using MATLAB/Simulink and CarSim. In this section, two simulation cases are presented to verify the effectiveness of the proposed MPC path tracking controller. The vehicle parameters are presented in Table 4. The reference path was parameterized as the curvature profile shown in Figure 7 and the position profile shown in Figure 8 using the path generation method proposed in Refs. $[32,33]$.

\subsection{Case 1}

To verify the performance of the adaptive coordinated controller, the road adhesion coefficient of 0.85 and a constant vehicle speed of $69 \mathrm{~km} / \mathrm{h}$ were set in the simulation. The first MPC controller (AFS MPC) control input is the lateral force of the front tires, for which the simulation results are represented by black dotted lines in the following figures, and those of the adaptive coordinated controller (AFS + DYC MPC) by blue solid lines.

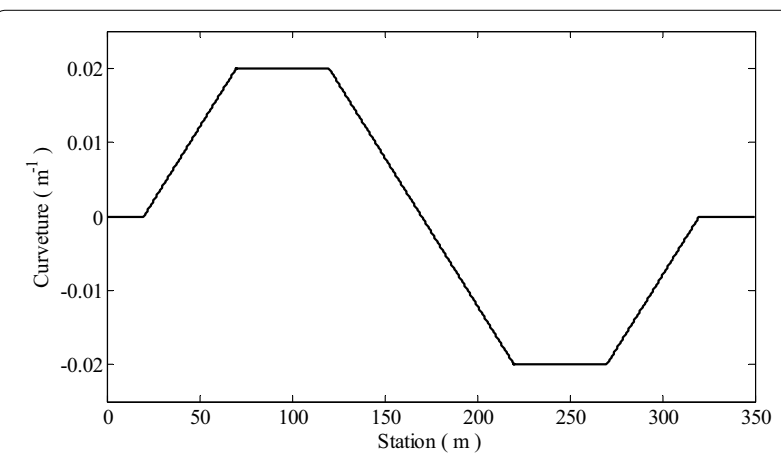

Figure 8 Reference path curvature

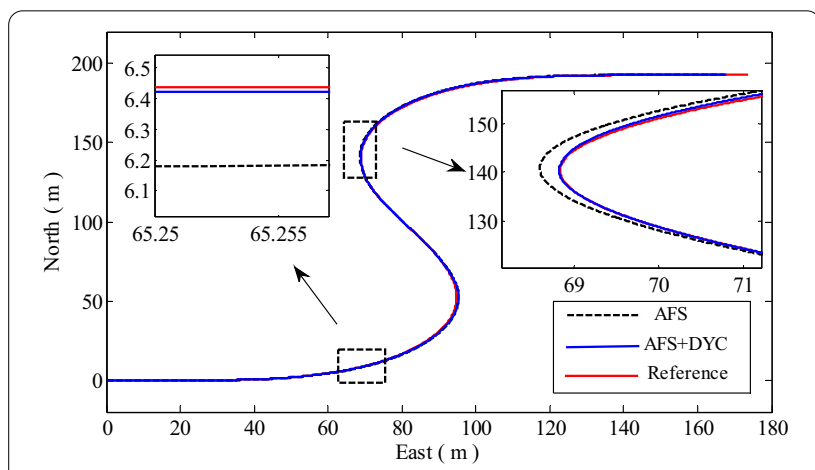

Figure 9 Global path

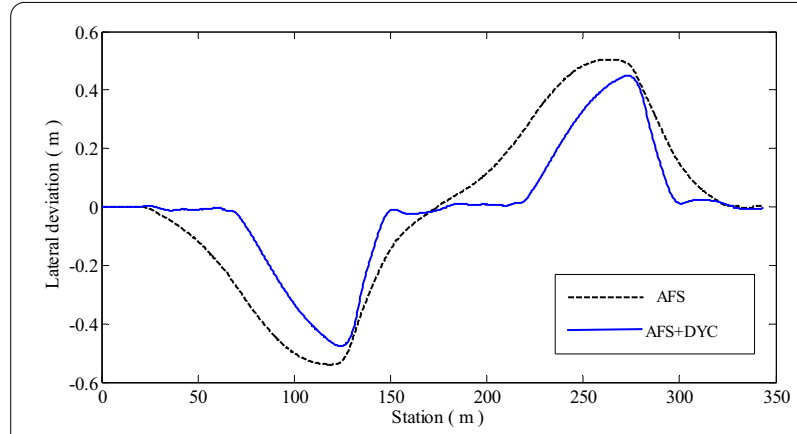

Figure 10 Lateral deviation

As can be seen from Figure 9, the controllers performed well under high-speed and large-curvature conditions. The path generated by the proposed controller is closer to the reference path (represented by the red solid line). Although the tracking performance decreased at larger curvatures, the proposed controller still achieved better control performance.

As can be seen from Figures 10 and 11 and Tables 5 and 6 , the absolute value of the maximum value of the lateral deviation $(\operatorname{Max} \operatorname{abs}(e))$ is reduced by $13.0 \%$, and that of 


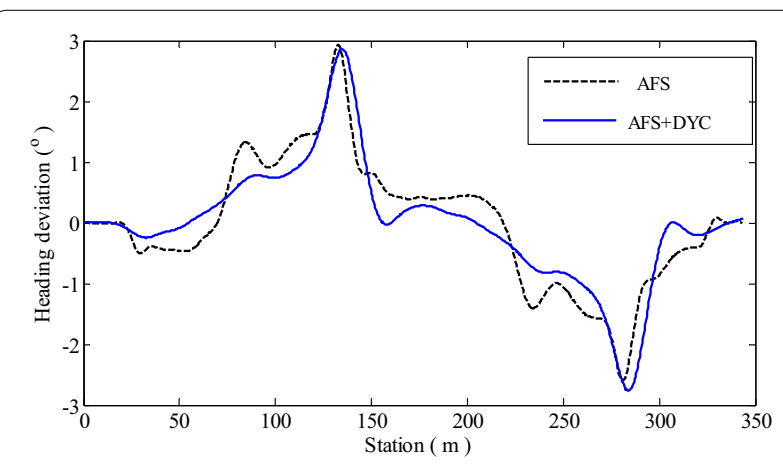

Figure 11 Heading deviation

Table 5 Lateral deviation comparison

\begin{tabular}{llll}
\hline Controllers & AV abs $(e)(m)$ & $\operatorname{MSE} \operatorname{abs}(e)(m)$ & $\operatorname{Max} \operatorname{abs}(e)(m)$ \\
\hline AFS & 0.23 & 0.04 & 0.54 \\
AFS + DYC & 0.13 & 0.03 & 0.47 \\
\hline
\end{tabular}

Table 6 Heading deviation comparison

\begin{tabular}{llll}
\hline Controllers & $\operatorname{AV} \operatorname{abs}\left(\theta_{e}\right)\left({ }^{\circ}\right)$ & $\operatorname{MSE} \operatorname{abs}\left(\theta_{e}\right)\left({ }^{\circ}\right)$ & $\operatorname{Max} \operatorname{abs}\left(\theta_{e}\right)\left({ }^{\circ}\right)$ \\
\hline AFS & 0.80 & 0.008 & 2.60 \\
AFS + DYC & 0.64 & 0.01 & 2.74 \\
\hline
\end{tabular}

the average value $(\mathrm{AV}$ abs $(e))$ reduced by $43.5 \%$ for the proposed controller compared to the AFS controller, and the former has a smaller absolute value of the mean square error (MSE abs $(e)$ ). For the heading deviation, although $\operatorname{AV} \operatorname{abs}\left(\theta_{e}\right)$ is decreased by $20.0 \%, \operatorname{Max} \operatorname{abs}\left(\theta_{e}\right)$ is increased by $4.2 \%$.

Compared with the AFS controller, the peak heading angle of the proposed controller appeared later. To avoid sudden changes, the yaw moment changed linearly when the proposed controller exited, which caused the peak of the heading angle deviation to be delayed with a relatively larger $\operatorname{Max} \operatorname{abs}\left(\theta_{e}\right)$ value of $2.74^{\circ}$. Therefore, the proposed controller is more effective for path tracking under highspeed and large-curvature conditions.

As shown in Figures 12, 13, 14, both controllers could ensure vehicle stability during path tracking. The maximum vehicle sideslip angle did not exceed $3^{\circ}$, the yaw rate did not exceed $5 \mathrm{rad} / \mathrm{s}$, and the maximum vehicle roll angle did not exceed $3^{\circ}$. The proposed controller provides better vehicle stability, especially in terms of the peak values of the vehicle sideslip angle, roll angle and the yaw rate.

Figures 15 and 16 show the relationships between the external yaw moment $(M)$, tire lateral force $\left(F_{y}\right)$, steady

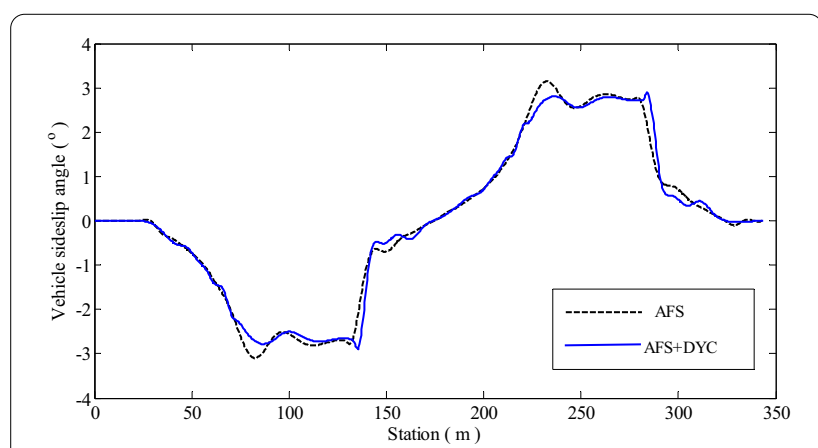

Figure 12 Sideslip angle

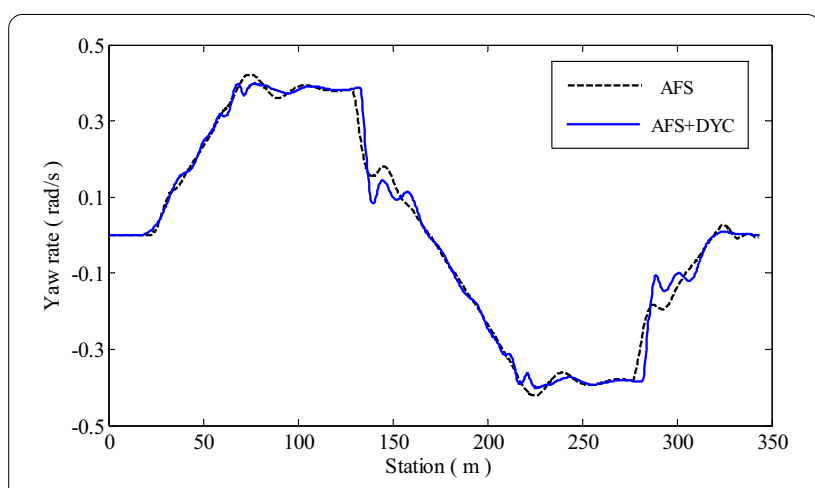

Figure 13 Yaw rate

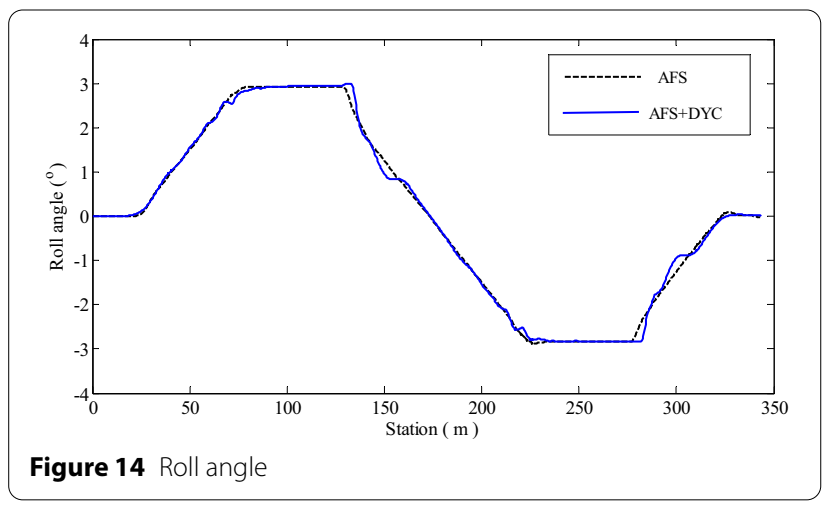

state vehicle lateral acceleration multiplied by 1000 $\left(1000 \times a_{y}^{s s}\right)$, actual vehicle lateral acceleration multiplied by $1000\left(1000 \times a_{y}\right)$, and path (Station). The proposed adaptive coordinated control strategy of active front wheel steering and direct yaw moment was initiated at positions 1 and 2 when the tire lateral force and vehicle acceleration satisfied the switching rules. The external yaw moment changed linearly at position 3 at the exit of the adaptive control strategy.

As shown in Figure 17, the observed value of the tire cornering stiffness was zero at the initial moment. This 


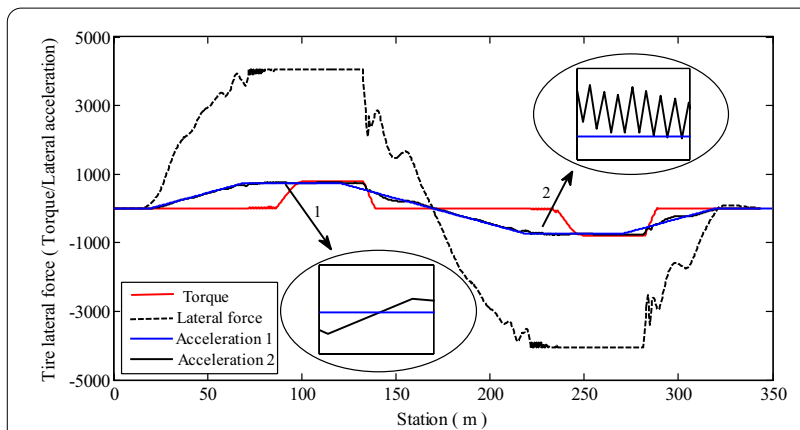

Figure 15 Coordinated control switching performance (DYC stepped in)

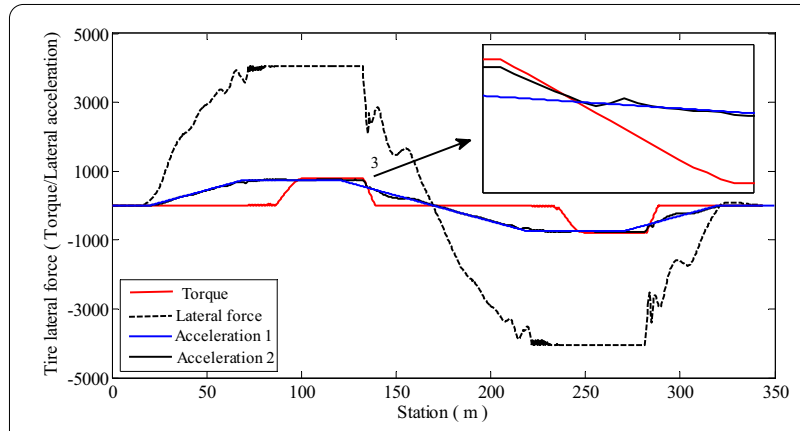

Figure 16 Coordinated control switching performance (DYC exit out)

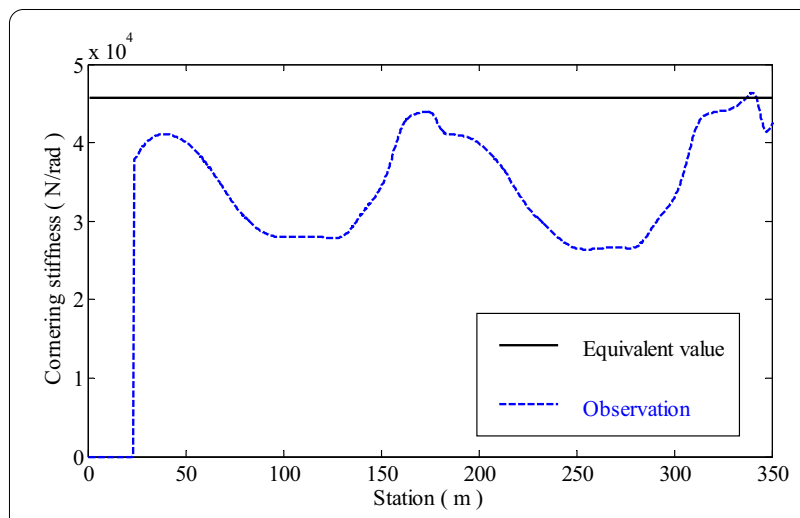

Figure 17 Cornering stiffness of rear tire

is because the data samples for the recursive matrices $\boldsymbol{M}$ and $\boldsymbol{N}$ at the initial moment were small and the random error was large, which resulted in large fluctuations in the observation results. The observation output value was therefore defined as 0 . When the sample data reached 100 groups, the matrices $\boldsymbol{M}$ and $\boldsymbol{N}$ were updated. Near the curvature peak of the reference path, the tire

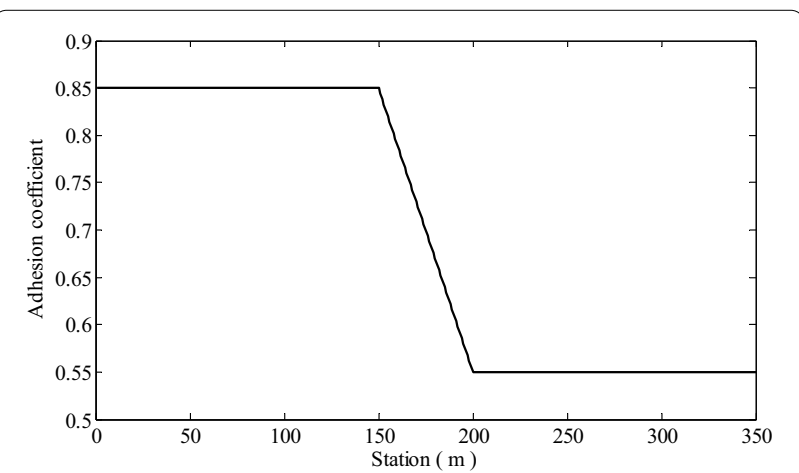

Figure 18 Road adhesion coefficient

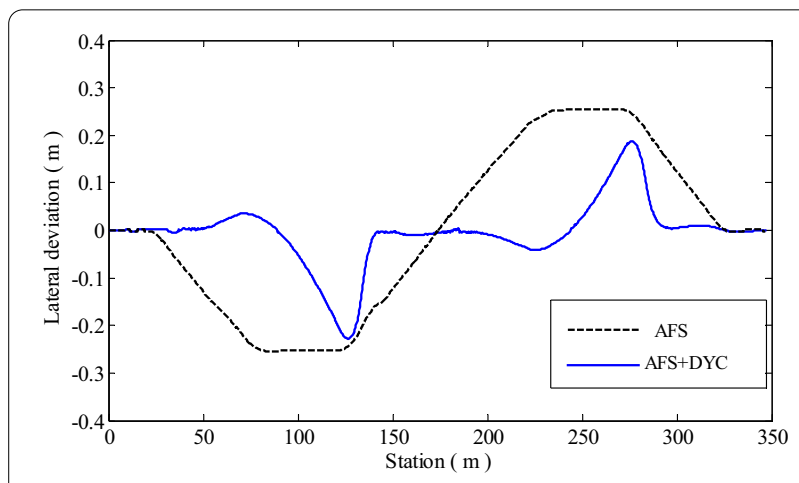

Figure 19 Lateral deviation

dynamics exhibited strong nonlinear characteristics, and the tire cornering stiffness reached its minimum peak value and remained stable. As the curvature gradually decreased, the observed value gradually converged to the equivalent cornering stiffness (constant value) of the linear tire model.

\subsection{Case 2}

To further verify the performance of the adaptive coordinated controller under extreme conditions, the vehicle speed was set to $57 \mathrm{~km} / \mathrm{h}$ and the nominal road adhesion coefficient to 0.85 . The actual road adhesion coefficient is shown in Figure 18. The reference path and its curvature are shown in Figures 7 and 8, respectively. The MPC controller (AFS MPC) control input was the lateral force of the front tires. The simulation results for AFS MPC are represented by black dotted lines, and those for the adaptive coordinated controller (AFS + DYC MPC) by blue solid lines in Figures 18, 19, 20.

As can be seen from Figures 19 and 20, the absolute values of the maximum lateral deviations are 0.25 and 0.22 for the AFS and the proposed controller, respectively. The absolute value of the maximum value was reduced in the latter by $12.0 \%$. The proposed controller 


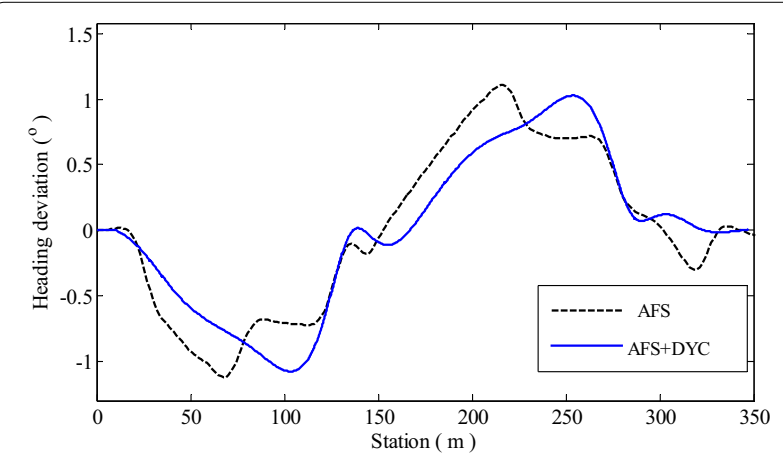

Figure $\mathbf{2 0}$ Heading deviation

exhibited better tracking performance. In particular, the superiority of the proposed controller in the areas of the road with low adhesion and large curvature are more significant when there are uncertainties in the road adhesion coefficient. The peak heading angle of the heading deviation appeared later for the proposed controller compared to the AFS controller. This is because the yaw moment changed linearly at the exit of the proposed controller to avoid sudden changes, which caused the peak of the heading angle deviation to be delayed and have a relatively large value. Therefore, the proposed controller is more effective for path tracking under high-speed and large-curvature conditions with uncertain values of the road adhesion coefficient.

As shown in Figures 21 and 22, both controllers could ensure vehicle stability during path tracking. The maximum vehicle sideslip angle did not exceed $0.70^{\circ}$, and the yaw rate did not exceed $0.35^{\circ} / \mathrm{s}$. However, the local stability performance of the AFS controller was slightly better than that of the proposed controller, as shown in Figures 21 and 22. This is because when the vehicle was on the peak curvature portion of the road, the vehicle gradually entered into the turning steady state (the vehicle sideslip angle and yaw rate tended to stabilize) during

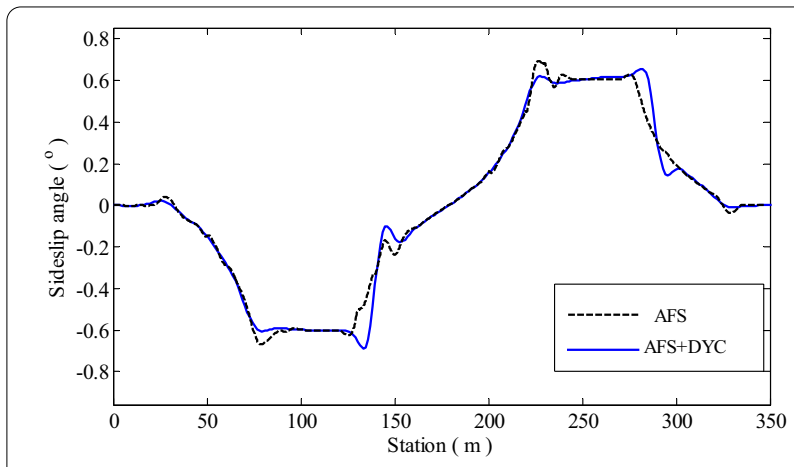

Figure 21 Sideslip angle

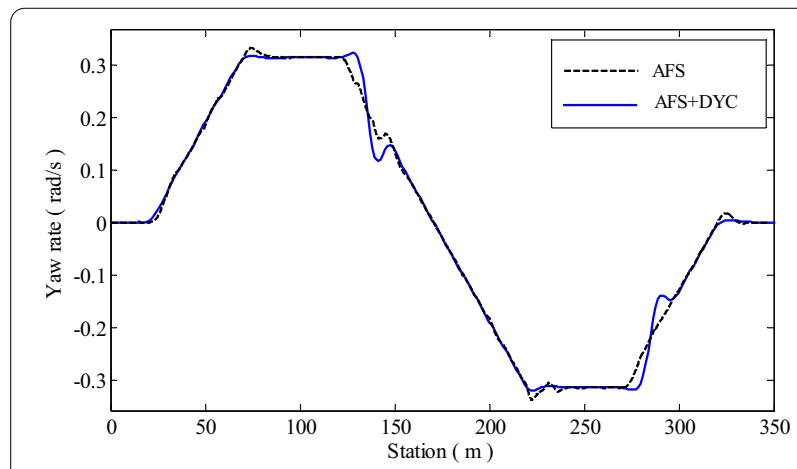

Figure $\mathbf{2 2}$ Yaw rate

the process of path tracking. At this time, DYC began to come into effect, which resulted in changes to the vehicle input and fluctuations in the vehicle stability indicators. To overcome these fluctuations, constraints were added to the change in yaw moment constraints at the moment of yaw moment intervention and withdrawal in this study. During the design process of the AFS and proposed controllers, the anti-instability constraints were constructed using the vehicle sideslip angle and yaw rate to ensure vehicle lateral stability. Therefore, vehicle stability under both strategies can be guaranteed, as can be verified from the results shown in Figures 21 and 22.

\section{Conclusions}

(1) In this study, the state-space model of the path tracking control system was established based on the vehicle dynamics, tire dynamics, and path tracking error models which are used to predict the system states in the prediction horizon.

(2) To improve the tracking accuracy and ensure vehicle stability under high-speed and large-curvature conditions, an adaptive coordinated path tracking control strategy was proposed. Under the strategy, the system prediction model is updated through tire parameter identification, the weight coefficient of the objective cost function is changed using fuzzy rules, and adaptive coordination between path tracking and DYC is realized.

(3) A path tracking controller was designed based on linear time-varying model predictive control algorithm that can systematically deal with vehicle stability constraints and control variable constraints.

(4) The results of the joint CarSim and Simulink simulation test showed that the adaptive coordinated control strategy is feasible. The control strategy can not only improve the path tracking accuracy but also ensure vehicle stability. Nevertheless, because 
parameter uncertainties, model errors, and external disturbances were not considered, a robust coordinated control strategy and hardware-in-the-loop test will be further studied in the future.

\section{Acknowledgements \\ Not applicable.}

\section{Authors' Contributions}

YT participated in the discussion of the control scheme and simulation. QY proposed the adaptive coordinated strategy and wrote this manuscript. PH revised this manuscript and participated in the discussion of simulation. SW analyzed the data and modified this manuscript. All authors read and approved the final manuscript.

\section{Authors' Information}

Ying Tian, born in 1974, is currently an associate professor at Beijing Jiaotong University, China. She received her PhD degree from Harbin Engineering University, China, in 2002. Her research interests include intelligent vehicle, man-machine system, hydrogen fuel cell and fault diagnosis.

Qiangqiang Yao, born in 1992, is currently a PhD candidate at Beijing Jiaotong University, China. He received his master degree from Shihezi University, China, in 2018. His research interests include vehicle dynamics and control, path tracking and robust control.

Peng Hang, born in 1991, is currently a research fellow at Nanyang Technological University, Singapore. He received his PhD degree from Tongji University, China, in 2018. His research interests include vehicle dynamics and control for driver-assistance systems, decision making, motion planning and motion control of autonomous vehicles.

Shengyuan Wang, born in 1997, is currently a master candidate at Beijing Jiaotong University, China.

\section{Funding}

Supported by the Foundation of Key Laboratory of Vehicle Advanced Manufacturing, Measuring and Control Technology (Beijing Jiaotong University), Ministry of Education, China (Grant No. 014062522006), National Key Research Development Program of China (Grant No. 2017YFB0103701).

\section{Competing Interests}

The authors declare no competing financial interests.

\section{Author Details}

${ }^{1}$ Beijing Key Laboratory of Powertrain for New Energy Vehicle, School of Mechanical, Electronic and Control Engineering, Beijing Jiaotong University, Beijing 100044, China. ${ }^{2}$ School of Mechanical and Aerospace Engineering, Nanyang Technological University, Singapore 639798, Singapore.

Received: 6 April 2021 Revised: 24 November 2021 Accepted: 8 December 2021

Published online: 04 January 2022

\section{References}

[1] P Song, B L Gao, S G Xie, et al. Optimal predictive control for path following of a full drive-by-wire vehicle at varying speeds. Chinese Journal of Mechanical Engineering, 2017, 30(3): 711-172.

[2] Q Q Yao, Y Tian, Q Wang, et al. Control strategies on path tracking for autonomous vehicle: state of the art and future challenges. IEEE Access, 2020, 8: 161211-161222.

[3] SB Xu, H Peng. Design, Analysis, and Experiments of preview path tracking control for autonomous vehicles. IEEE Transactions on Intelligent Transportation Systems, 2020, 21(1): 48-58.

[4] P Hang, XXia, XChen. Handling stability advancement with 4WS and DYC coordinated control: a gain-scheduled robust control approach. IEEE Transactions on Vehicular Technology, 2021, 70(4): 3164-3174.
[5] Q Q Yao, Y Tian. A model predictive controller with longitudinal speed compensation for autonomous vehicle path tracking. Applied Sciences, 2019, 9(22): 4739.

[6] F Lin, Y W Zhang, Y Q Zhao, et al. Trajectory tracking of autonomous vehicle with the fusion of DYC and longitudinal-lateral. Chinese Journal of Mechanical Engineering, 2019, 32: 16.

[7] CY Sun, X Zhang, L H Xi, et al. Design of a path-tracking steering controller for autonomous vehicles. Energies, 2018, 11(6): 1451.

[8] P Hang, X B Chen, W Wang. Cooperative control framework for human driver and active rear steering system to advance active safety. IEEE Transactions on Intelligent Vehicles, 2021, 6(3): 460-469.

[9] CY Sun, X Zhang, Q Zhou, et al. A model predictive controller with switched tracking error for autonomous vehicle path tracking. IEEE Access, 2019, 7: 53103-53114.

[10] A T Nguyen, C Sentouh, H Zhang, et al. Fuzzy static output feedback control for path following of autonomous vehicles with transient performance improvements. IEEE Transactions on Intelligent Transportation Systems, 2020, 21(7): 3069-3079.

[1 1] Z J Wang, J M Wang. Ultra-local model predictive control: A model-free approach and its application on automated vehicle trajectory tracking. Control Engineering Practice, 2020, 101: 104482.

[12] W Zhang. A robust lateral tracking control strategy for autonomous driving vehicles. Mechanical Systems and Signal Processing, 2021, 150: 107238.

[13] M Fnadi, W Q Du, F Plumet, et al. Constrained model predictive control for dynamic path tracking of a bi-steerable rover on slippery grounds. Control Engineering Practice, 2021. 107: 104693.

[14] K Kritayakirana, J C Gerdes. Using the center of percussion to design a steering controller for an autonomous race car. Vehicle System Dynamics, 2012. 50(suppl.1): 33-51.

[15] N R Kapania, J C Gerdes. Design of a feedback-feedforward steering controller for accurate path tracking and stability at the limits of handling. Vehicle System Dynamics, 2015, 53(12): 1687-1704.

[16] J Funke, M Brown, S M Erlien, et al. Collision avoidance and stabilization for autonomous vehicles in emergency scenarios. IEEE Transactions on Control Systems Technology, 2017, 25(4): 1204-1216.

[17] S M Erlien, S Fujita, J C Gerdes. Shared steering control using safe envelopes for obstacle avoidance and vehicle stability. IEEE Transactions on Intelligent Transportation Systems, 2016, 17(2): 441-451.

[18] JY Goh, T Goel, J C Gerdes. Toward automated vehicle control beyond the stability limits: drifting along a general path. Journal of Dynamic Systems, Measurement, and Control, 2020: 142.

[19] A Nahidi, A Kasaiezadeh, S Khosravani, et al. Modular integrated longitudinal and lateral vehicle stability control for electric vehicles. Mechatronics, 2017, 44: 60-70

[20] A Tahouni, M Mirzaei, B Najjari. Novel constrained nonlinear control of vehicle dynamics using integrated active torque vectoring and electronic stability control. IEEE Transactions on Vehicular Technology, 2019, 68(10): 9564-9572.

[21] H N Peng, W D Wang, Q An, et al. Path tracking and direct yaw moment coordinated control based on robust MPC with the finite time horizon for autonomous independent-drive vehicles. IEEE Transactions on Vehicular Technology, 2020, 69(6): 6053-6066.

[22] J C Chen, Z B Shuai, H Zhang, et al. Path following control of autonomous four-wheel-independent-drive electric vehicles via second-order sliding mode and nonlinear disturbance observer techniques. IEEE Transactions on Industrial Electronics, 2021, 68(3): 2460-2469.

[23] J H Guo, Y G Luo, K Q Li, et al. Coordinated path-following and direct yaw-moment control of autonomous electric vehicles with sideslip angle estimation. Mechanical Systems and Signal Processing, 2018, 105: 183-199.

[24] J Xie, XXu, FWang, et al. Coordinated control based path following of distributed drive autonomous electric vehicles with yaw-moment control. Control Engineering Practice, 2021, 106: 104659.

[25] C Hu, H Jing, R R Wang, et al. Robust output-feedback control for path following of autonomous ground vehicles. Mechanical Systems and Signal Processing, 2016, 70-71: 414-427.

[26] HTaghavifar, S Rakheja. Path-tracking of autonomous vehicles using a novel adaptive robust exponential-like-sliding-mode fuzzy type-2 neural network controller. Mechanical Systems and Signal Processing, 2019, 130: $41-55$.

[27] J Zhang, B J Zhang, N Zhang, et al. A novel robust event-triggered fault tolerant automatic steering control approach of autonomous land 
vehicles under in-vehicle network delay. International Journal of Robust and Nonlinear Control, 2021, 31(7): 2436-2464.

[28] L Ling, M Li, M Yu, et al. Parameter identification method for the tire cornering stiffness of model vehicle. Autimotive Engineering, 2016, 38(12): 1508-1515. (In Chinese)

[29] F Lin, H D Zhang, Y Q Zhao, et al. Road friction condition identification based on tire lateral stiffness estimation. Journal of South China University of Technology, 2019, 47(11): 16-24. (in Chinese)

[30] Y Tian, Q Q Yao, C Q Wang, et al. Switched model predictive controller for path tracking of autonomous vehicle considering rollover stability. Vehicle System Dynamics, 2021. https://doi.org/10.1080/00423114.2021.1999990.

[31] P G Stankiewicz, A A Brown, S N Brennan. Preview horizon analysis for vehicle rollover prevention using the zero-moment point. Journal of Dynamic Systems, Measurement, and Control, 2015, 137: 091002.

[32] X H Li, Z P Sun, D P Cao, et al. Development of a new integrated local trajectory planning and tracking control framework for autonomous ground vehicles. Mechanical Systems and Signal Processing, 2017, 87: 118-137.

[33] G Pereira, L Svensson, Lima, et al. Lateral model predictive control for over-actuated autonomous vehicle. Proceedings of 2017 IEEE Intelligent Vehicles Symposium, Redondo Beach, CA, USA, June 11-14, 2017 : 310-316.

\section{Submit your manuscript to a SpringerOpen ${ }^{\circ}$ journal and benefit from:}

- Convenient online submission

- Rigorous peer review

- Open access: articles freely available online

- High visibility within the field

- Retaining the copyright to your article

Submit your next manuscript at $\gg$ springeropen.com 